\title{
An Expansion Method Dealing with Spatial Incompleteness of Measured Mode Shapes of Beam Structures
}

\author{
Liu Fushun $^{1, *}$, Chen Wenwen ${ }^{1}$ and Wang Weiying ${ }^{2}$ \\ ${ }^{1}$ College of Engineering, Ocean University of China, China \\ ${ }^{2}$ Center for Engineering Test and Appraisal, Qingdao Technological University, China
}

Received: 27 Mar. 2013, Revised: 28 Jul. 2013, Accepted: 30 Jul. 2013

Published online: 1 Mar. 2014

\begin{abstract}
Obtaining mode shape values at rotational degrees of freedom (dofs) is a challenge from dynamic test of beam structures, for the reason that rotational dofs are usually difficult to be measured. This article proposes an expansion method to deal with spatial incompleteness of measured mode shapes of beam structures. One improvement is that the constrained linear least squares is combined into traditional expansion procedure proposed by Liu (2011), to estimate modeling errors in a physical meaning interval; and the initial guesses on mode-correction coefficients could be obtained using a non-iterative technique for improving the convergence of the approach. A cantilever beam is used to investigate the proposed method by establishing two numerical models: one is a beam finite element model as a baseline; the other is the model to represent real structure with different stiffness distribution compared with the baseline. One can conclude from numerical results that the approach has a better convergence performance than traditional expansion method, and spatially incomplete measured modes for beam structures, including higher order modes, can be expand properly and to be fundamental modal data for model updating or damage detection of beam structures.
\end{abstract}

Keywords: Expansion, Spatial incompleteness, Beam structure, Mode shape

\section{Introduction}

Though beam structures are wide used in engineering, it very difficult to obtain rotational mode shape values in experiment because of the limitations of present test devices. The mismatch between the measured mode shape and the corresponding one from finite element model should be solved firstly for the application of model updating and damage detection in structures [1,2].

In general, there are two techniques solving spatial incompleteness of measured mode shapes: one is model reduction, and the other is modal expansion. Guyan [3] and Irons [4] firstly proposed static reduction and expansion method, by partitioning the mass and stiffness matrices into a set of master and slave dofs. This method is wide used because it can provide accurate results for lower order mode shapes, while errors will increase with the order of mode shapes increase. In addition, the type and number of master dofs will have a significant influence on model reduction or modal expansion. For improving Guyan's method, inertial terms were taken into account when estimate slave dofs from measured master dofs $[5,6]$, while these improved methods also have to be selected carefully. O'Callahan [7] proposed the System-Equivalent-Reduction Expansion Process (SEREP) method, and this method has long been recognized to be more accurate than Guyan scheme in matching modal data for both modal expansion and model reduction. One should note that it is on the opposite when the experimental mode shapes are not well correlated with the corresponding analytical one [8]. Liu (2011) [9] presented an iterative method for dealing with spatial incompleteness of measured experimental mode shapes. While estimation of model-correction factors in a physical meaningful interval is not discussed. Liu and $\mathrm{Li}$ (2013) [10] discussed the application of an iterative expansion method to offshore platforms, though the problem of model errors' estimation in a physical meaningful interval is studied, how to improve convergence performance become unsolved. Reference [11] studied a non-iterative mode shape expansion

\footnotetext{
*Corresponding author e-mail: percyliu@ouc.edu.cn
} 
technique, numerical and experimental results demonstrated the effectiveness of the proposed approach. The limitation is that discrepancy between the test model and the corresponding finite element model should in a certain range.

\section{Preliminary: Least squares formulations}

In mathematics, a bound constrained least squares technique could be used to solve inverse problem, and variables in a mathematical model can be estimated in prescribed intervals by

$$
\min \|\mathbf{A x}-\mathbf{b}\|_{2}^{2}, \alpha \leq x \leq \beta
$$

Where $x$ is a variable vector, $A$ and $b$ is coefficient matrix respectively, and $\alpha, \beta$ are corresponding bound conditions.

When $A$ is not full rank, a popular approach is Tikhonov regularization [12]

$$
\min \|\mathbf{A x}-\mathbf{b}\|_{2}^{2}+\lambda^{2}\left\|\mathbf{L}\left(\mathbf{x}-\mathbf{x}_{0}\right)\right\|_{2}^{2}
$$

where $x_{0}$ is an initial parameter estimate and $L$ is typically chosen to yield approximations to the $l$ th order derivative, $l=0,1,2 . \lambda$ is called the regularization parameter which can be calculated using Generalized Cross-Validation (GCV), the Discrepancy principle, and so on [13].

\section{Modeling Errors Estimation Based on the Improvement of initial guess on mode-correction factors}

As studied in reference [9], the model-correction factors and the mode-correction factors are coupled in the following equation

$$
\left(\mathbf{K}_{n, i j}^{\diamond}+\delta_{j}^{T} \tilde{\mathbf{K}}_{i j}^{s}\right) \alpha=\mathbf{f}-\left(\mathbf{K}_{s, i j}^{\diamond}-\mathbf{M}_{s, i j}^{\diamond}\right) \delta_{j}
$$

Where $K_{n, i j}^{\diamond}, \tilde{K}_{i j}^{s}, K_{s, i j}^{\diamond}$ and $M_{n, i j}^{\diamond}$ are the $N m$-by- $N e, N$ by- $N e, N m$-by- $N$ and $N m$-by- $N$ matrices, respectively, $\alpha$ and $\delta$ are model-correction factors and mode-correction factors respectively.

To solve Eq. (3), the possible method is to provide an initial guess on mode-correction factors $\delta$, which will have a significant influence on the iterative solution of Eq. (3). In the following, our main purpose is to find better initial guesses on $\delta$.

Contrast to initial values in reference [9]-these values are obtained from the finite element model directly, we make an improvement on these initial values based on measured modal information, by neglecting the modeling errors, as expressed by

$$
\mathbf{K} \Phi_{j}{ }^{\prime}-\lambda_{j}{ }^{\prime} \mathbf{M} \Phi_{j}{ }^{\prime}=0
$$

Where $K$ and $M$ are stiffness and mass matrices of the finite element model, $\lambda_{j}^{\prime}, \Phi_{j}^{\prime}$ are the jth measured eigenvalues and eigenvectors of the true structure. Eq. (4) is based on the assumption that

$$
K=K^{\prime}
$$

$$
M=M^{\prime}
$$

Eq. (5) and Eq. (6) show that modeling errors between the finite element model and the true model are ignored.

Using $\Phi_{i}^{T}$ to represent eigenvectors of the ith order from finite element model, then premultiplying Eq. (4), one obtains

$$
\Phi_{i}^{T} \mathbf{K} \Phi_{j}{ }^{\prime}-\lambda_{j}{ }^{\prime} \Phi_{i}^{T} \mathbf{M} \Phi_{j}{ }^{\prime}=0
$$

Assume the jth mode $\Phi_{j}^{\prime}$ is a modification of $\Phi_{j}^{\prime \prime}$ by

$$
\Phi_{j}^{\prime}=\Phi_{j}^{\prime \prime}+\sum_{s=1}^{N} \delta_{S} \Phi_{s, j}^{\prime \prime}
$$

Where

$$
\Phi_{j}^{\prime \prime}=\left\{\begin{array}{c}
\phi_{m} \\
\phi_{m} \\
\vdots \\
1 \\
1 \\
\vdots \\
1
\end{array}\right\}
$$

Substitute Eq. (8) and Eq. (9) into Eq. (7), one obtains

$$
\begin{aligned}
& \Phi_{i}^{T} \mathbf{K} \Phi^{\prime \prime}{ }_{j}+\sum_{s=1}^{N} \delta_{s} \Phi_{i}^{T} \mathbf{K} \Phi^{\prime \prime}{ }_{s, j}- \\
& \left(\lambda_{j}{ }^{\prime} \Phi_{i}^{T} \mathbf{M} \Phi^{\prime \prime}{ }_{j}+\lambda_{j}{ }^{\prime} \sum_{s=1}^{N} \delta_{s} \Phi_{i}^{T} \mathbf{M} \Phi^{\prime \prime}{ }_{s, j}\right)=0
\end{aligned}
$$

Denote

$$
\begin{gathered}
\mathrm{H}_{i j}^{\Theta}=\Phi_{i}^{T} \mathbf{K} \Phi^{\prime}{ }_{j} \\
\mathrm{H}_{s, i j}^{\Theta}=\Phi_{i}^{T} \mathbf{K} \Phi^{\prime}{ }_{s, j} \\
\mathrm{E}_{i j}^{\Theta}=\lambda_{j}^{*} \Phi_{i}^{T} \mathbf{M} \Phi^{\prime}{ }_{j} \\
\mathrm{E}_{s, i j}^{\Theta}=\lambda_{j}^{*} \Phi_{i}^{T} \mathbf{M} \Phi^{\prime}{ }_{s, j}
\end{gathered}
$$

Thus Eq. (10) can be rewritten as

$$
\mathrm{H}_{i j}^{\Theta}+\sum_{s=1}^{N} \delta_{s, j} \mathrm{H}_{s, i j}^{\Theta}=\lambda_{j}^{*} \mathrm{E}_{i j}^{\Theta}+\lambda_{j}^{*} \sum_{s=1}^{N} \delta_{s, j} \mathrm{E}_{s, i j}^{\Theta}
$$

Rearranging Eq. (15), yields

$$
\sum_{s=1}^{N} \delta_{s, j}\left(K_{s, i j}^{\Theta}-\lambda_{j}^{*} M_{s, i j}^{\Theta}\right)=\lambda_{j}^{*} \mathrm{E}_{i j}^{\Theta}-\mathrm{H}_{i j}^{\Theta}
$$


Letting

$$
\begin{gathered}
\mathbf{G}=\mathrm{H}_{s, i j}^{\Theta}-\lambda_{j}^{*} \mathrm{E}_{s, i j}^{\Theta} \\
\Delta_{j}^{0}=\left\{\begin{array}{c}
\delta_{1} \\
\delta_{2} \\
\vdots \\
\delta_{N}
\end{array}\right\}
\end{gathered}
$$

Eq. (16) can be rewritten as

$$
\mathbf{G} \Delta_{j}^{0}=\lambda_{j}^{*} \mathrm{E}_{i j}^{\Theta}-\mathrm{H}_{i j}^{\Theta}
$$

Analytically, Eq. (19) can be solved using a standard inverse operation if $G$ is a nonsingular square matrix,

$$
\Delta_{j}^{0}=\mathbf{G}^{-1}\left(\lambda_{j}^{*} \mathrm{E}_{i j}^{\Theta}-\mathrm{H}_{i j}^{\Theta}\right)
$$

Substituting Eq. (20) into Eq. (3), then Eq. (3) can be uncoupled

$$
\left(\mathbf{K}_{n, i j}^{\diamond}+\Delta_{j}^{0^{T}} \tilde{\mathbf{K}}_{i j}^{s}\right) \alpha=\mathbf{f}-\left(\mathbf{K}_{s, i j}^{\diamond}-\mathbf{M}_{s, i j}^{\diamond}\right) \Delta_{j}^{0}
$$

Similar to Eq. (2.1), Eq. (3.19) can be solved employing the bound constrained least squares technique

$$
\min \|\mathbf{A} \alpha-\mathbf{b}\|_{2}^{2}, \mathbf{P}_{1} \leq \alpha \leq \mathbf{P}_{2}
$$

Where $P_{1}$ and $P_{2}$ define a physical meaningful interval for modeling errors' estimation, and

$$
\begin{gathered}
\mathrm{A}=\left(\mathbf{K}_{n, i j}^{\diamond}+\Delta_{j}^{0^{T}} \tilde{\mathbf{K}}_{i j}^{s}\right) \\
\mathbf{b}=\mathbf{f}-\left(\mathbf{K}_{s, i j}^{\diamond}-\mathbf{M}_{s, i j}^{\diamond}\right) \Delta_{j}^{0}
\end{gathered}
$$

\section{Numercial Study}

A cantilever beam as shown in Figure 1, is used to investigate the proposed method by establishing two numerical models: one is a beam model as a baseline whose stiffness and mass denoted as $K$ and $M$; the other is the model to represent real structure with different stiffness distribution compared with the baseline, this model could be called the measured model or true model, and denoted as $K^{\prime}, M^{\prime}$ respectively. The length of the beam is $4 \mathrm{~m}$; Young's modulus is taken as $3.2 \times 10^{10} \mathrm{~Pa}$, and mass density per unit length $125 \mathrm{~kg} / \mathrm{m}$. The cross section area and the moment of inertia are $0.05 \mathrm{~m}^{2}$ and $1.66 \times 10^{-4} \mathrm{~m}^{4}$, respectively.

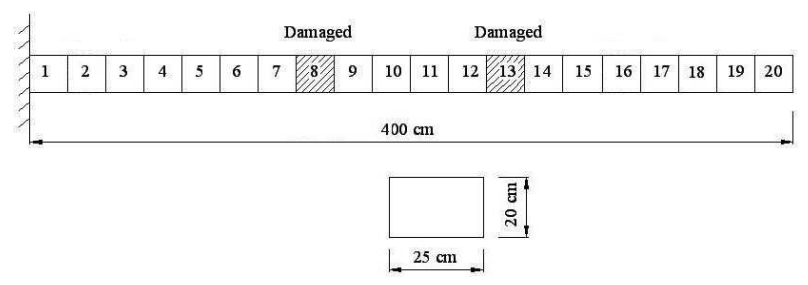

Fig. 1: A cantilever beam to be measured
A real structure with different stiffness distribution is established by regarding it as a damaged beam considering the deterioration of stiffness. Here, two damages are modeled by reducing stiffness at elements 8 and 13 by $35 \%$ and $55 \%$, respectively, from the baseline model. Besides stiffness reduction at above two damaged elements, the parameters of the true model are considered to be slightly different from those of the baseline model for other elements too. Specifically, the modeling errors of other elements of the true model are produced based on the absolute value of the Gaussian distribution, with mean 0 and standard deviation 0.05 . The first five frequencies of the true model are 6.954, 41.90, 117.32, 243.47 and $379.45 \mathrm{~Hz}$, respectively.

Firstly, we demonstrate the correctness and the feasibility of the approach. Because each node has 2 dofs, translational and rotational dofs, then we can calculate the dofs of the beam $2 \times 20=40$. Assume all dofs of the beam are measured, and mode-correction factors are known and boundary conditions are imposed as $-1 \leq \alpha \leq 0$. Implementing the improved method without iteration, one obtains Fig. 2, which demonstrates that model correction factors could be estimated perfectly.

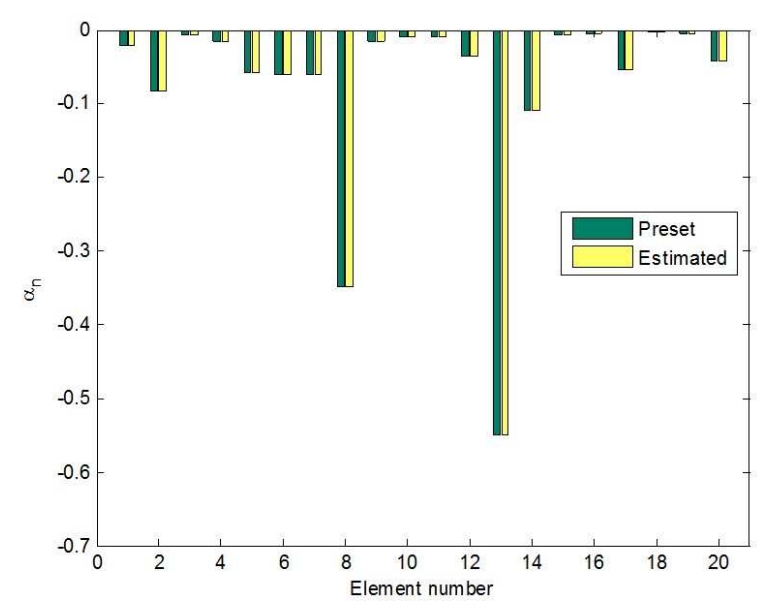

Fig. 2: Comparison of preset and estimated model correction factors

Convergence performance of the approach will be investigated in the following discussion. As discussed in reference [9], the difference between the true and estimated mode shape values at master dofs was employed to evaluate the performance of convergence, and denoted as $\kappa$. Assume translational dofs could be measured and the first five modes are expected to be expanded, which means 20 rotational dofs are taken as slave ones and should be estimated. Implementing our proposed approach and traditional technique in reference [9] with 5 iterations, a comparison of normalized $\kappa$ is 
shown in Fig. 3. One can find significant improvement on convergence performance utilizing our approach, which means iteration numbers in our proposed method could be reduced when applied to complex structures. A comparison of estimated model correction factors employing traditional expansion method and our approach imposing the constraint $-0.6 \leq \alpha \leq 0$ is shown in Fig. 4. From Fig. 4 one can conclude that our proposed method could estimate modeling errors in a certain interval and provide more accurate estimation results, which also mean a physical meaningful range is easy and effective to be imposed.

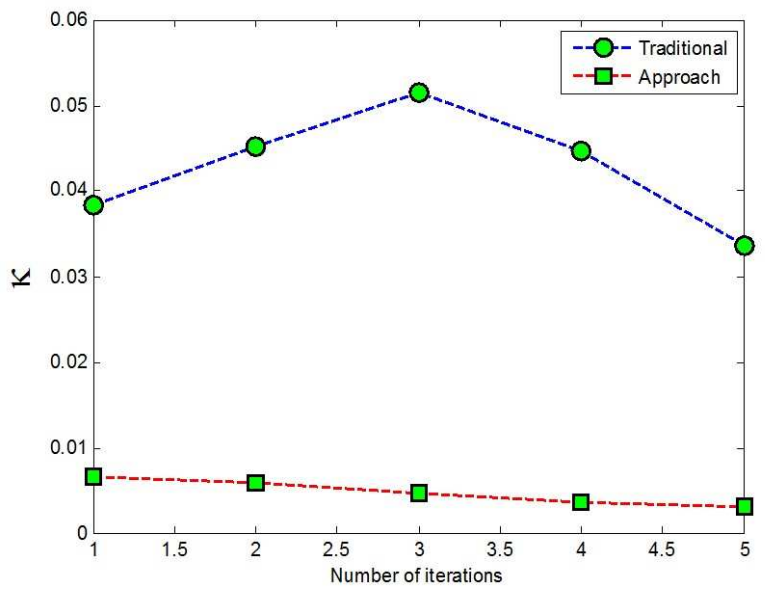

Fig. 3: $\kappa$ against the number of iterations employing traditional method and our approach

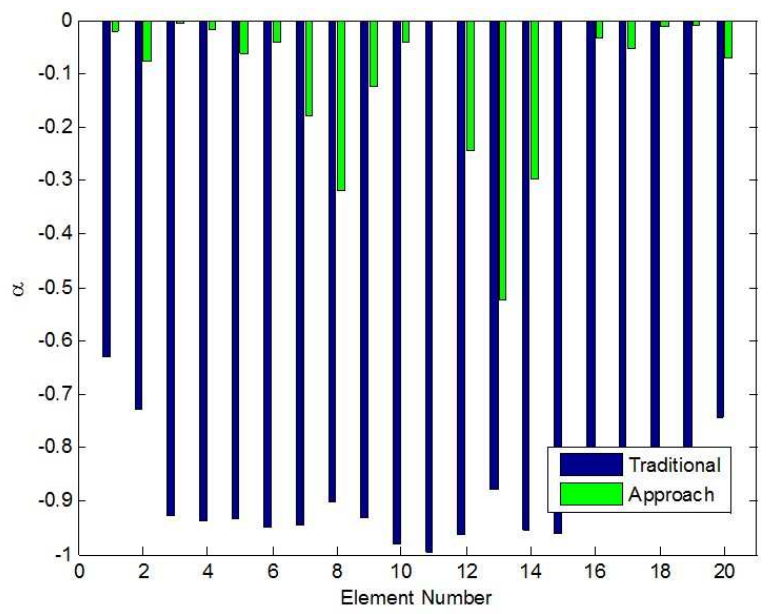

Fig. 4: Comparison of estimated model correction factors employing traditional expansion method and our approach
Table 1: MAC values from the existing methods and the improved approach

\begin{tabular}{ccccc}
\hline \multirow{2}{*}{ Mode } & \multicolumn{4}{c}{ MAC } \\
\cline { 2 - 5 } & Guyan & Dynamic & Serep & Improved \\
\hline 1 & 1 & 1 & 0.99999 & 1 \\
2 & 0.99993 & 0.99993 & 0.99951 & 0.99998 \\
3 & 0.99974 & 0.99974 & 0.99878 & 0.99994 \\
4 & 0.99994 & 0.99994 & 0.99976 & 0.99996 \\
5 & 0.99894 & 0.99895 & 0.99433 & 0.99983 \\
\hline
\end{tabular}

Then, we compare the performance of mode shape expansion from the approach to that of present techniques, such as Guyan, Dynamic, and SEREP method. In this example, modeling errors are considered as discussed above, the purpose of the mode shape expansion not only includes modeling errors' estimation in a physical interval, but also with better MAC values between the true (simulated) spatially complete mode shapes and expanded one. Assume the constraint on modeling errors is $-1 \leq \alpha \leq 0$, and implementing the proposed method, one can obtain MAC values of the first five mode shape between the true and the expanded as 1 , $0.99998,0.99994,0.99996$ and 0.99983 , respectively; detailed comparison results between Guyan, Dynamic, SEREP and the approach are shown in Table 1. Fig. 5 to Fig. 7 is the first three mode shapes comparison results at slave dofs using the Guyan method and the proposed approach, respectively. The numerical results indicate that these modes can be expanded well and modeling errors can be estimated reasonably in a physical meaningful range.

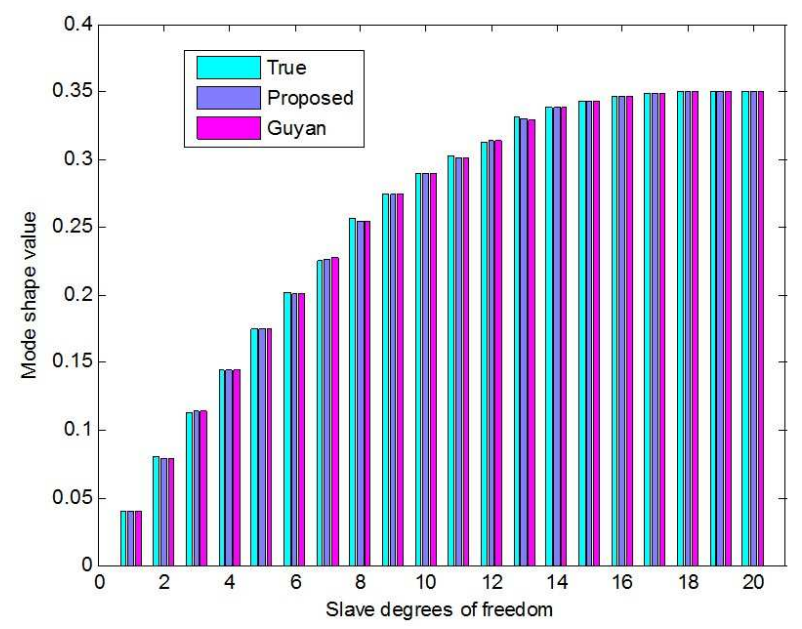

Fig. 5: The first mode shape comparison at slave dofs using the existing methods and the proposed approach 


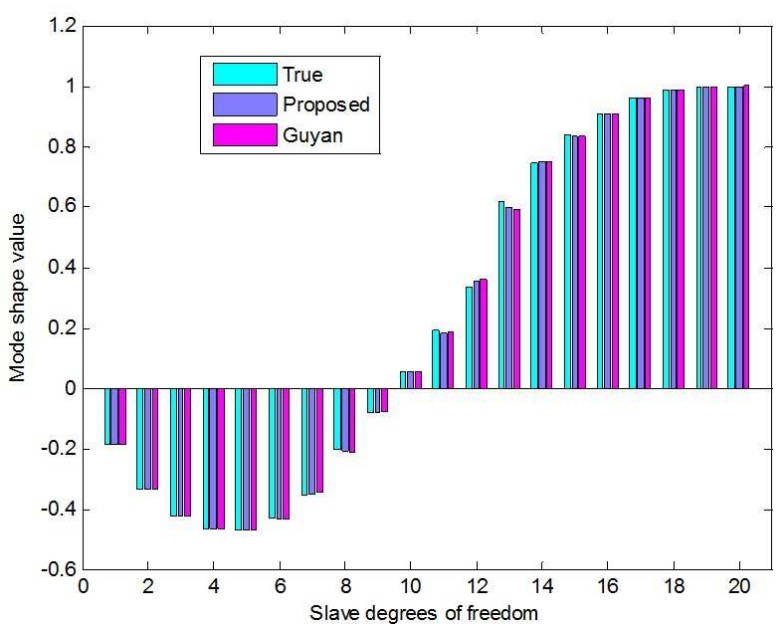

Fig. 6: The second mode shape comparison at slave dofs using the existing methods and the proposed approach

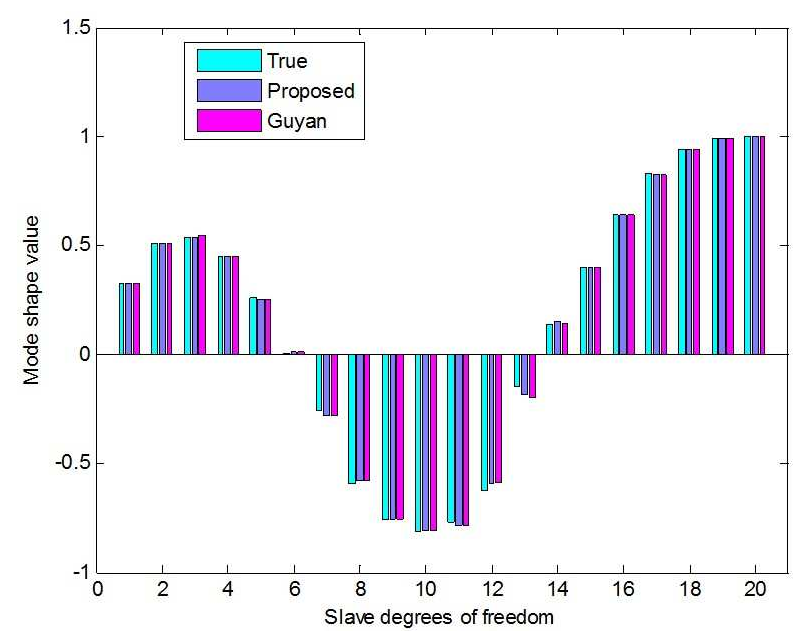

Fig. 7: The third mode shape comparison at slave dofs using the existing methods and the proposed approach

\section{Conclusion}

In practice, it is impossible for model-correction factors less than -1 , as discussed in reference [9] because when this factor equal to -1 which means the completely loss of stiffness; even the member of the structure has been strengthened, we also can conclude that the factor should be in a certain interval. This article proposes an expansion method to deal with the spatial incompleteness of measured mode shapes of beam structures. One improvement is that the constrained linear least squares is combined into traditional expansion procedure proposed by Liu (2011), to estimate modeling errors in a physical meaning interval; and the initial guesses on mode-correction coefficients could be obtained using a non-iterative technique for improving the convergence of the approach. Numerical results from the cantilever beam show that 1) modeling errors could be estimated in a physical meaningful interval using constrained least squares effectively, 2) the approach outperforms traditional mode shape expansion method in convergence performance, and 3) the proposed method could expand spatially incomplete measured modes of beam structures properly, especially for higher order modes.

\section{Acknowledgement}

The authors acknowledge the financial support of the 973 project, Grant no. 2011CB013704, of the National Natural Science Foundation of China, Grant nos. $51279188,51009124$.

\section{References}

[1] J. E. Mottershead and M. I. Friswell, Journal of Sound and Vibration, 167, 347-375 (1993).

[2] H. P. Chen and N. Bicanic, Computational Mechanics, 37, 455-467 (2006).

[3] R. J. Guyan, AIAA Journal, 3, 380 (1965).

[4] B. Irons, AIAA Journal, 3, 961- 962 (1965).

[5] R. L. Kidder, AIAA Journal, 11, 892 (1973).

[6] C.A. Miller, Journal of the Structural Division, 106, 20972108 (1980).

[7] J. C. O'Callahan, P. Avitabile, R. Riemer, Proceeding of Seventh International Modal Analysis Conference, 29-37 (1989).

[8] H. J. Li, F. S. Liu, S-L J. Hu, Science in China Series E: Technological Sciences, 51, 2254-2268 (2008).

[9] F. S. Liu, Journal of Sound and Vibration, 330, 4633-4645 (2011).

[10] F. S. Liu, and H. J. Li, Ocean Engineering, 63, 26-34 (2013).

[11] F. S. Liu, and H. J. Li, Ocean Engineering, 51, 119-128 (2012) .

[12] A. N. Tikhonov, Soviet Math, 4, 1624-1627 (1963).

[13] P. C. Hansen, Numer. Algorithms, 6, 1-35 (1994). 


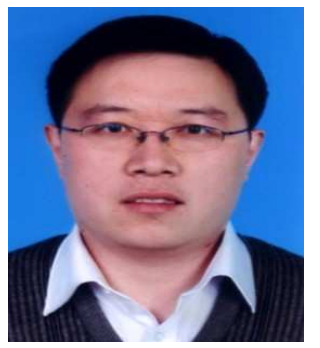

Liu Fushun received the $\mathrm{PhD}$ degree in port, coastal and offshore engineering from Ocean University of China, He is currently an Associate Professor with the Department of engineering at Ocean University of China, China. He has published 48 papers in peer reviewed journals and proceedings of conference. His research interests are in the areas of dynamic analysis of ocean engineering, including model updating, damage detection, damping identification.

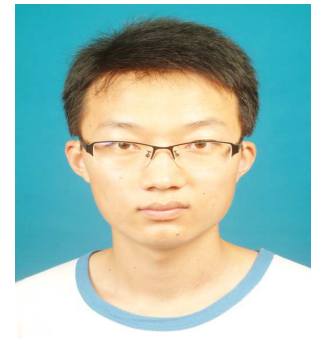

engineering.

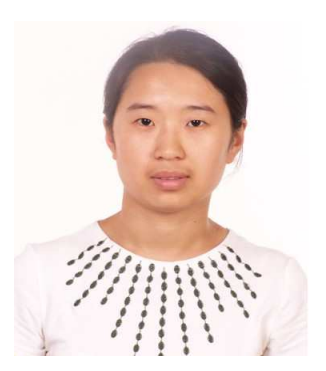

Chen Wenwen received the BC degree from Ocean University of China, he is currently a student in port, coastal and offshore engineering for MS Degree in Ocean University of China. His research interests are in the areas of structural dynamic analysis of offshore

Wang Weiying received the MS degree in engineering structure from Qingdao Technological University, She is currently a Civil Engineer at Qingdao Technological University, China. Her research interests are in the areas of Engineering Test and Appraisal of civil structures. 